Association for Information Systems AIS Electronic Library (AISeL)

Wirtschaftsinformatik Proceedings 2003

Wirtschaftsinformatik

September 2003

\title{
Eignung multimedialer Lernobjekte zur Erreichung der in Blooms Taxonomie unterschiedenen Lernziele
}

Gerhard F. Knolmayer

Universität Bern, gerhard.knolmayer@iwi.unibe.ch

Corinne Montandon

Universität Bern

Follow this and additional works at: http://aisel.aisnet.org/wi2003

\section{Recommended Citation}

Knolmayer, Gerhard F. and Montandon, Corinne, "Eignung multimedialer Lernobjekte zur Erreichung der in Blooms Taxonomie unterschiedenen Lernziele" (2003). Wirtschaftsinformatik Proceedings 2003. 43.

http://aisel.aisnet.org/wi2003/43

This material is brought to you by the Wirtschaftsinformatik at AIS Electronic Library (AISeL). It has been accepted for inclusion in Wirtschaftsinformatik Proceedings 2003 by an authorized administrator of AIS Electronic Library (AISeL). For more information, please contact elibrary@aisnet.org. 
In: Uhr, Wolfgang, Esswein, Werner \& Schoop, Eric (Hg.) 2003. Wirtschaftsinformatik 2003: Medien - Märkte - Mobilität, 2 Bde. Heidelberg: Physica-Verlag

ISBN: 3-7908-0111-9 (Band 1)

ISBN: 3-7908-0116-X (Band 2)

(c) Physica-Verlag Heidelberg 2003 


\section{Eignung multimedialer Lernobjekte zur Erreichung der in Blooms Taxonomie unterschiedenen Lernziele}

\section{Gerhard F. Knolmayer, Corinne Montandon}

Universität Bern

Zusammenfassung: In diesem Beitrag fassen wir die im angelsächsischen Raum viel beachtete Taxonomie von Bloom zusammen, die sechs Klassen von Lernzielen unterscheidet. Wir stellen sodann Multimedia-Lernobjekte aus dem Bereich der Logistik dar, die am Institut für Wirtschaftsinformatik der Universität Bern für Projekte im Virtuellen Campus Schweiz und der Virtual Global University entwickelt wurden und ordnen diese Lernobjekte den Klassen von Blooms Taxonomie $z u$. Dabei zeigt sich, dass sich die Multimedia-Objekte zur Erreichung aller von Bloom formulierten Lernziele beitragen können. Daraus folgern wir, dass für eine virtuelle Lehre Einsatzmöglichkeiten auch zur Erreichung anspruchsvoller Lernziele und-inhalte bestehen.

Schlüsselworte: Multimedia, e-Learning, Lernziele, Blooms Taxonomie

\section{Einleitung}

In Verbindung mit web-basiertem Lernen hat die Entwicklung von multimedialen Lernobjekten in den letzten Jahren erheblich an Bedeutung gewonnen. Ein Multimedia-Objekt (MMO) ist interaktiv manipulierbar und kann bei Vorliegen bestimmter Bedingungen auf Ereignisse mit Aktionen reagieren, die auf dem Einsatz von Methoden beruhen [vgl. Schu02, S. 28; für weitere Definitionen vgl. z. B. GrMe97, S. 6 ff]. Ein MMO verwendet mehrere Formen der Kommunikation mit dem Lernenden; dazu können Text, Zahlen, Grafiken, Ton, Musik, Bilder, Film und Software gehören. Einige Merkmale von MMO sind [vgl. Schu02, S. 22]:

- Die Daten aus verschiedenen Medien werden integriert und von einem Rechner verarbeitet.

- Dem Lernenden kann Multimedia einen multisensorischen Eindruck vermitteln, wobei eine bestimmte Information mehrfach mittels verschiedener Medien repräsentiert werden kann.

- Zwischen MMO und Benutzern sind Interaktionen möglich. 
Von verschiedenen Spezifikationskonsortien und Standardisierungsgremien wird zur Erhöhung der Wiederverwendung von Lerninhalten deren Strukturierung in e-Learning Objekte (ELO) [vgl. z. B. Wile02; Knol03] gefordert. Ein für Lernumgebungen entwickeltes Multimedia-Objekt kann als Multimediales Lernobjekt (MLO) bezeichnet werden.

Im folgenden Abschnitt fassen wir die von Bloom et al. entwickelte und vor allem in angelsächsischen Bereich viel beachtete Taxonomie von Lernzielen zusammen. In Abschnitt 3 stellen wir verschiedene MLO aus dem Bereich der Logistik dar, die im Rahmen zweier e-Learning-Projekte am Institut für Wirtschaftsinformatik der Universität Bern entwickelt wurden. Diese wurden durch den Swiss Virtual Campus' bzw. das Bundesministerium für Wissenschaft und Forschung im Rahmen von Projekten der Virtual Global University (VGU) ${ }^{2}$ gefördert. Sodann überprüfen wir, für die Erreichung welcher Lernziele sich die skizzierten MLO eignen

\section{Blooms Taxonomie von Lernzielen}

1956 publizierte ein Komitee von College und University Examiners aus dem US Bundesstaat Michigan eine sechs Elemente umfassende Taxonomie von kognitiven Lernzielen. Dieses Handbuch wurde von Benjamin S. Bloom herausgegeben [Bloo $\left.{ }^{+} 56\right]$ und in der Folge ist "Blooms Taxonomie" jedenfalls im angelsächsischen Sprachraum sehr bekannt geworden; im deutschen Sprachraum fand sie hingegen wenig Beachtung (Tabelle 1).

\begin{tabular}{|c|c|}
\hline Suchbegriff & Zahl der Treffer mit Google \\
\hline Bloom taxonomy & 52400 \\
\hline "Bloom's taxonomy" & 21600 \\
\hline Taxonomie Bloom & 1400 \\
\hline Taxonomie Bloom (Seiten auf Deutsch) & 381 \\
\hline Blooms Taxonomie (Seiten auf Deutsch) & 44 \\
\hline "Blooms Taxonomie" (Seiten auf Deutsch) & 15 \\
\hline
\end{tabular}

Tabelle 1: Zahl der Quellen am Web [2003-05-13]

1 Projekt OPESS (Operations Management, ERP and SCM Systems), gefördert u.a. durch den Virtuellen Campus Schweiz

http://opess.ie.iwi.unibe.ch/; http://www.virtualcampus.ch/

2 Kurs "ERP and Beyond" im Rahmen des MBI-Programms der Virtual Global University, gefördert u.a. durch das Projekt "Neue Medien in der Bildung" des Bundesministeriums für Bildung und Forschung

http://www.vg-u.de/; http://www.medien-bildung.net
Die kognitiven Lernziele betreffen den Erwerb und den Wiederaufruf von Wissen und die Entwicklung intellektueller Fähigkeiten; in diesem Bereich hat die intensivste Auseinandersetzung in der Curriculumentwicklung stattgefunden und die Lernziele wurde am klarsten definiert. Bloom und seine Kollegen schlagen folgende Klassifikation der kognitiven Lernziele vor [Bloo ${ }^{+} 56$, S. 201]:

1. "Knowledge ... involves the recall of specifics and universals, the recall of methods and processes, or the recall of a pattern, structure, or setting. For measurement purposes, the recall situation involves little more than bringing to mind the appropriate material."

Dieser Bereich wird unterteilt in

1.1 Knowledge of Specifics, in dem die Reproduzierbarkeit von spezifischen und isolierbaren Einzelinformationen betrachtet wird

1.2 Knowledge of Ways and Means of Dealing with Specifics, womi ein mittlerer Abstraktionsgrad angesprochen wird. Im Zentrum steht weniger die Aktivität des Lernenden in der Verwendung der Materialien als das passive Wissen um ihre Beschaffenheit.

1.3 Knowledge of the Universals and Abstractions in a Field, worunter die übergeordneten Schemata und Muster verstanden werden, nach denen Phänomene und Ideen organisiert werden. Dazu gehören Verallgemeinerungen und Theorien, die beim Studium der Phänomene oder Problemlösungen angewandt werden.

2. Intellectual Abilities and Skills "... refer to organized modes of operation and generalized techniques for dealing with materials and problems. ... the abilities and skills objectives emphasize the mental processes of organizing and reorganizing material to achieve a particular purpose." Dieses Fähigkeiten werden auch als Comprehension bezeichnet und als niedrigste Ebene des Verstehens bezeichnet: "It refers to a type of understanding or apprehension such that the individual knows what is being communicated and can make use of the material or idea being communicated without necessarily relating it to other material or seeing its fullest implications" [Bloo ${ }^{+} 56$, S. 204]. Dieser Bereich wird unterteilt in

2.1 Translation, die beurteilt wird auf der Basis von Wahrhaftigkeit und Genauigkeit, also der Bewahrung der Inhalte des ursprünglichen Materials trotz Änderung der Sprache oder Kommunikationsform.

2.2 Interpretation, in der das Material erklärt oder zusammengefasst wird.

2.3 Extrapolation, in der Trends und Tendenzen über die gegebenen Daten hinaus verwendet werden, um u.a. Implikationen, Konsequenzen und Effekte zu bestimmen. 
3. Application, wobei Abstraktionen in bestimmten, konkreten Situationen verwendet werden. Diese können die Form von allgemeinen Ideen, prozeduralen Regeln oder verallgemeinerten Methoden annehmen, aber auch die Erinnerung und Anwendung technischer Prinzipien, Ideen und Theorien umfassen.

4. Analysis wird umschrieben als "... breakdown of a communication into its constituent elements or parts such that the relative hierarchy of ideas is made clear and/or the relations between the ideas expressed are made explicit. Such analyses are intended to clarify the communication, to indicate how the communication is organized, and the way in which it manages to convey its effects, as well as its basis and arrangement" [Bloo ${ }^{+} 56, \mathrm{~S}$. 205]. Unterschieden wird zwischen der

4.1 Analyse von Elementen

4.2 Analyse von Beziehungen

4.3 Analyse von Organisationsprinzipien, wobei sowohl explizite als auch implizite Strukturen betrachtet werden.

5. Synthese, in der die Elemente und Teile zu einem Ganzen zusammengefügt werden. In diesem Prozess soll ein Muster oder eine Struktur entstehen, die zuvor nicht klar sichtbar gewesen ist. Diese Bereich wird unterteilt in die

5.1 Erarbeitung von Material, mit dem versucht wird, Ideen, Gefühle und/oder Erfahrungen anderen zugänglich zu machen.

5.2 Erarbeitung eines Plans oder eines Vorschlags für Vorgehensweisen, wobei die Anforderungen der Aufgabe entweder vorgegeben oder vom Lernenden selbst entwickelt werden.

5.3 Ableitung einer Menge abstrakter Beziehungen, um bestimmte Phänomene oder Daten zu klassifizieren oder zu erläutern oder eine Deduktion von Propositionen und Beziehungen aus einer Menge elementarer Propositionen oder symbolischer Repräsentationen.

6. Evaluation, in welcher der Wert von Materialien und Methoden im Hinblick auf bestimmte Ziele quantitativ oder qualitativ beurteilt werden soll. Die Ziele können vorgegeben oder vom Lernenden formuliert werden. Unterteilt wird dieser Bereich in

6.1 Judgements in terms of internal evidence, worunter diese unter Gesichtspunkten wie logischer Genauigkeit und Konsistenz beurteilt werden.

6.2 Judgements in terms of external criteria, in denen das Material u.a. im Hinblick auf erinnerte Gesichtspunkte beurteilt wird.
Blooms Taxonomie wurde in Verbindung mit e-Learning z. B. verwendet, um Inhalte am Web [Thib01] oder die Kommunikation zwischen Lernenden [WaRa99] zu klassifizieren.

\section{Darstellung ausgewählter multimedialer Lern- objekte in der Logistik-Ausbildung}

Im Folgenden werden fün MLO aus dem Bereich der Logistik-Ausbildung vorgestellt und im Hinblick auf die Taxonomie von Bloom klassifiziert. Wir prüfen, welche der in Blooms Taxonomie formulierten Lernziele der kognitiven Domain durch diese MLO unterstützt werden und versuchen auf diese Weise, die Mächtigkeit multimedialen Lernens besser einschätzen zu können. Die im Folgenden dargestellten MLO sind in Papierform nur beschränkt darstellbar; einen besseren Eindruck von ihren Inhalten und ihrer Dynamik vermittelt

http://www.ie.iwi.unibe.ch/wi2003/

\subsection{Multimediales Lernobjekt 1: Gozinto-Modell}

Insbesondere im deutschen Sprachraum wird die Vorgehensweise bei der Ableitung von Sekundärbedarfen aus Primärbedarfen gerne an Gozinto-Graphen veranschaulicht. Diese Bezeichnung geht auf Andrew Vazsonyi zurück, der 1954 behauptete, der berühmte italienische Mathematiker Zeparzet Gozinto habe ein neues Verfahren der Stücklistenauflösung entwickelt [Vazs54]. Dieser fiktive Name leitete sich aus einer Verballhornung des Satzes "The part that goes into" ab.

Die Regeln zur Bestimmung der Sekundärbedarfe sind einfach. Der Algorithmus läuft in mehreren Schritten $\mathrm{ab}$, wobei Konsistenzbedingungen hinsichtlich der Reihenfolge, in der die Berechnungen ausgeführt werden, einzuhalten sind (Berücksichtigung der Dispositionsstufen). Dies lässt sich in grafischen Darstellungen in Papierform nur beschränkt veranschaulichen. Wir haben daher ein MLO entwickelt, welches die Vorgehensweise am Gozinto-Graphen in Einzelschritte zerlegt, die der Studierende durch Anklicken auslöst. Er hat zudem die Möglichkeit, $\mathrm{zu}$ früheren Zuständen der Berechnung zurückzukehren. Durch die Farbgebung wird deutlich gemacht, welche Elemente des Gozinto-Graphen Gegenstand der aktuell ausgefuihrten Berechnungen sind.

In Abbildung 1 sind die Primärbedarfe in den (im MLO gelb gekennzeichneten) Quadraten an den Knoten 4, 5 und 8 angegeben. Für die Endprodukte 4 und 8 stimmen Primärbedarf und Gesamtbedarf überein. Im ersten Schritt könnte entweder der Gesamtbedarf des Teils 4 oder des Teils 8 aufgelöst werden. Wir unterstellen, dass der Lernende zunächst den Bedarf von Teil 4 auflöst. In Abbildung 1 
werden die durch Auflösung des Gesamtbedarfs von Teil 4 entstandenen Sekundärbedarfe von 300 und 100 an den Knoten 1 und 6 in grauen Feldern dargestellt. Im zweiten Schritt muss der Bedarf an Produkt 8 aufgelöst werden. Die daraus resultierenden Sekundärbedarfe werden an den Knoten 1, 5 und 3 mit 10, 40 und 20 (im MLO in roter Farbe) angegeben (Abbildung 1).

Für das Verständnis dieses MLO muss der Studierende Kenntnisse über die Vorgehensweisen bei der Materialbedarfsbestimmung besitzen. Die Berechnungen wurden in einer ersten Version des MLO nur für eine ganz bestimmte Datenkonstellation veranschaulicht. Der Studierende konnte in dieser Version den Algorithmus nicht auf eine andere vorgegebene oder eine von ihm gewählte Datenkonstellation anwenden. Im Sinn der Taxonomie von Bloom ist ein derartiges MLO jedenfalls der Kategorie "Knowledge" zuzuordnen; wir neigen zur Aussage, dass es auch Kategorie 2, "Comprehension", unterstützt. Eine selbstständige "Application" von Wissen liegt nicht jedoch nicht vor.

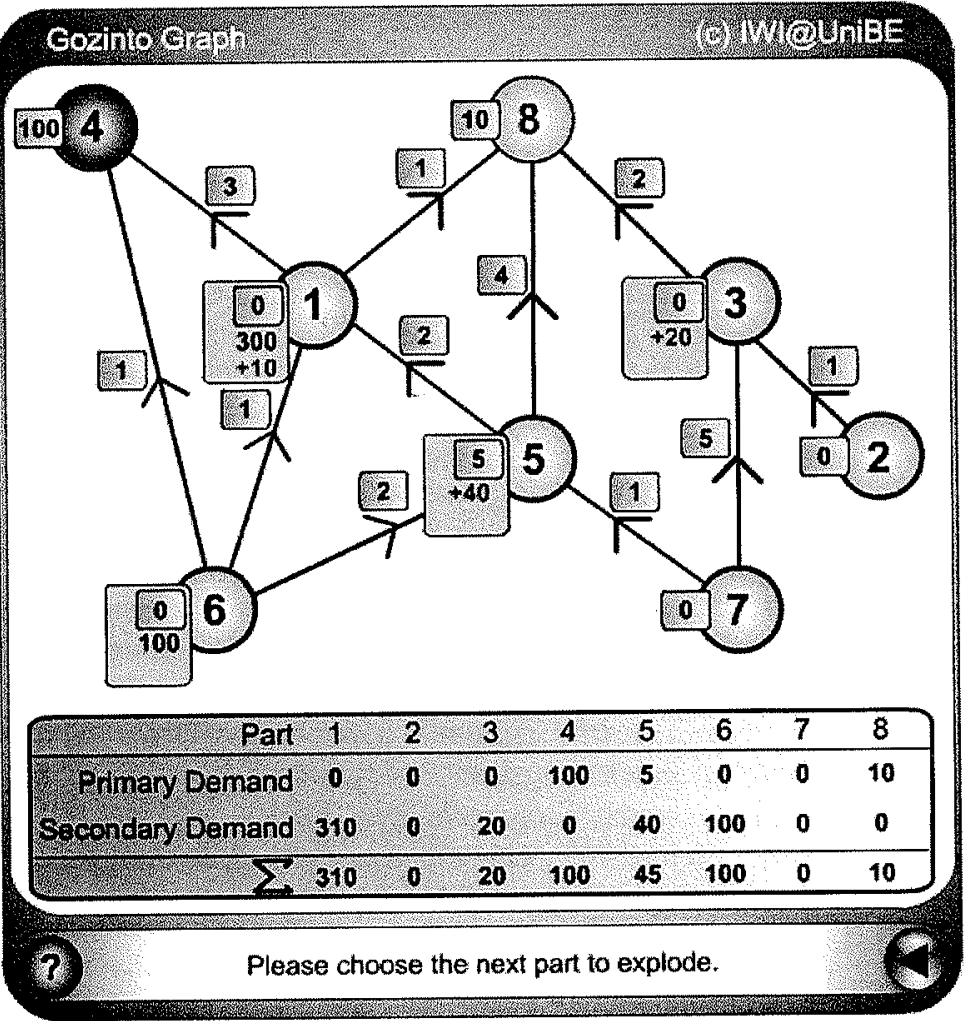

Abbildung 1: Gozinto-Modell nach Bedarfsauflösung für Teil 8
Mögliche Erweiterungen der oben beschriebenen MLO-Version sind:

a) Der Lernende muss durch Anklicken eines Kreises festlegen, welches Teil im nächsten Schritt aufgelöst werden soll. Diese Erweiterung ist der dritten, als "Application" bezeichneten Klasse der Taxonomie Blooms zuzuordnen.

b) Der Lernende kann selbst gewählte Daten für Stücklisten und Primärbedarfe innerhalb der vorgegebenen Teilestruktur definieren und damit seine Anwendungserfahrungen auf eine breitere Basis stellen.

c) Eine Datenkonstellation könnte so gestaltet sein, dass zumindest an einem Knoten des Gozinto-Graphen negative Gesamtbedarfe auftreten. Dies tritt ein, wenn zu hohe Werte für eine geplante Reduktion der Lagerbestände vorgegeben wurden. In diesem Fall sind die Berechnungen am Gozinto-Graphen so zu modifizieren, dass der negative Gesamtbedarf durch eine Null ersetzt wird. Ein derart erweitertes MLO deckt auch die vierte und fünfte Kategorie Blooms, die "Analyse" und "Synthese", ab.

d) Der Lernende kann aufgefordert werden, die Berechnung am GozintoGraphen durch Matrizenrechnung zu überprüfen. Er würde dann feststellen, dass die Vorgehensweisen im Normalfall zu gleichen Ergebnissen führen, im unter (c) erörterten Fall aber voneinander abweichen. Er könnte aufgefordert werden, diese Systemeigenschaften zu erklären und einen Vergleich der Vor- und Nachteile zwischen der Berechnung am Gozinto-Graphen und der Lösung durch Matrizenrechnung vorzunehmen. In diesem Fall würde das MLO zusätzlich eine Methodenevaluation fordern und damit der sechsten von Bloom definierten Klasse zuzuordnen sein.

Die beschriebenen Erweiterungen wurden in einem zweiten Schritt implementiert und sind unter

http://www.ie.iwi.unibe.ch/wi2003/

abrufbar.

\subsection{Multimediales Lernobjekt 2: Terminierte Bedarfsauf- lösung ("Brutto-Netto-Rechnung")}

In ERP- und PPS-Systemen wird die Bedarfsauflösung mehrperiodisch vorgenommen. Zur Veranschaulichung der Vorgehensweise müssten im Gozinto-Graphen die skalaren Bedarfswerte durch Bedarfsvektoren ersetzt werden. Bereits in einfachen Beispielen wird sowohl die grafische Veranschaulichung als auch die tabellarische Rechnung schwierig nachvollziehbar. 
Wir veranschaulichen dies an der von Scheer [Sche97, S. 142; vgl. auch Kurb99, S. $130 \mathrm{ff} ;$ Mert01, S. $148 \mathrm{f}]$ dargestellten "Brutto-Netto-Rechnung", die in Abbildung 2 wiedergegeben wird. Diesem Beispiel liegt der in Abbildung 3 rechts oben dargestellte Gozinto-Graph zu Grunde, an dessen Kanten Direktbedarfe und Vorlaufzeiten angegeben sind. Die Berechnungen erfordern subtile Überlegungen, deren Verständnis Studierenden oft Schwierigkeiten bereitet. Insbesondere muss in der Tabelle zwischen Eingabedaten und abgeleiteten Daten unterschieden werden. In der tabellarischen Darstellung ist schwierig zu vermitteln, welche Berechnungen in welcher Sequenz ausgeführt werden. Dazu kommt, dass der Aufbau der Tabelle aus Sicht der zu Grunde liegenden betriebswirtschaftlichen Sachverhalte verändert werden sollte.

In den Lehrveranstaltungen an der Universität Bern und der Virtual Global University verwenden wir eine etwas modifizierte Darstellung. Um die Logik der Berechnungen $\mathrm{zu}$ veranschaulichen, haben wir zu der von uns modifizierten Tabelle ein Viewlet ${ }^{3}$ entwickelt, das einzelne Tabelleneinträge erläutert (vgl. Abbildung 3). Wegen der Vergänglichkeit der Viewlet-Anzeigen stellen wir die in ihm verwendeten Erläuterungen zudem auf einer Help-Seite zum jederzeitigen Aufruf bereit. Ein von uns entwickeltes Java-Applet veranschaulicht die Reihenfolge der Berechnungen. Der Studierende kann z. B. Stücklisten- und Kostendaten sowie die Vorlaufzeiten (innerhalb der gegebenen Fertigungsstruktur und des vorgegebenen Planungshorizonts) verändern und die Berechnung für die veränderten Datenkonstellationen nachvollziehen

Dieses MLO ist interaktiv und verwendet Texte, farbig unterschiedene Zahlen sowie eine Grafik. Die in den Viewlets gegebenen Erläuterungen könnten auch akustisch vermittelt werden. Die an den Kanten des Gozinto-Graphen dargestellten Informationen über Stücklisten und Vorlaufzeiten werden automatisch den vom Studierenden vorgenommenen Eingaben angepasst. Das MLO fordert ein detailliertes Verständnis der Mechanismen der mehrperiodischen Bedarfsauflösung, sodass wir es den Kategorien 1 und 2 der Taxonomie von Bloom (Knowledge und Comprehension) zuordnen. Eine fachlich qualifizierte "Application" durch den Studierenden liegt nicht vor, weil er die Rechenregeln nicht selbstständig anwenden muss. Dies würde z. B. die Verwendung eines (möglicherweise vorstrukturierten) Excel-Blattes voraussetzen, in dem der Studierende die vorzunehmenden Berechnungen formelmäßig definieren muss.

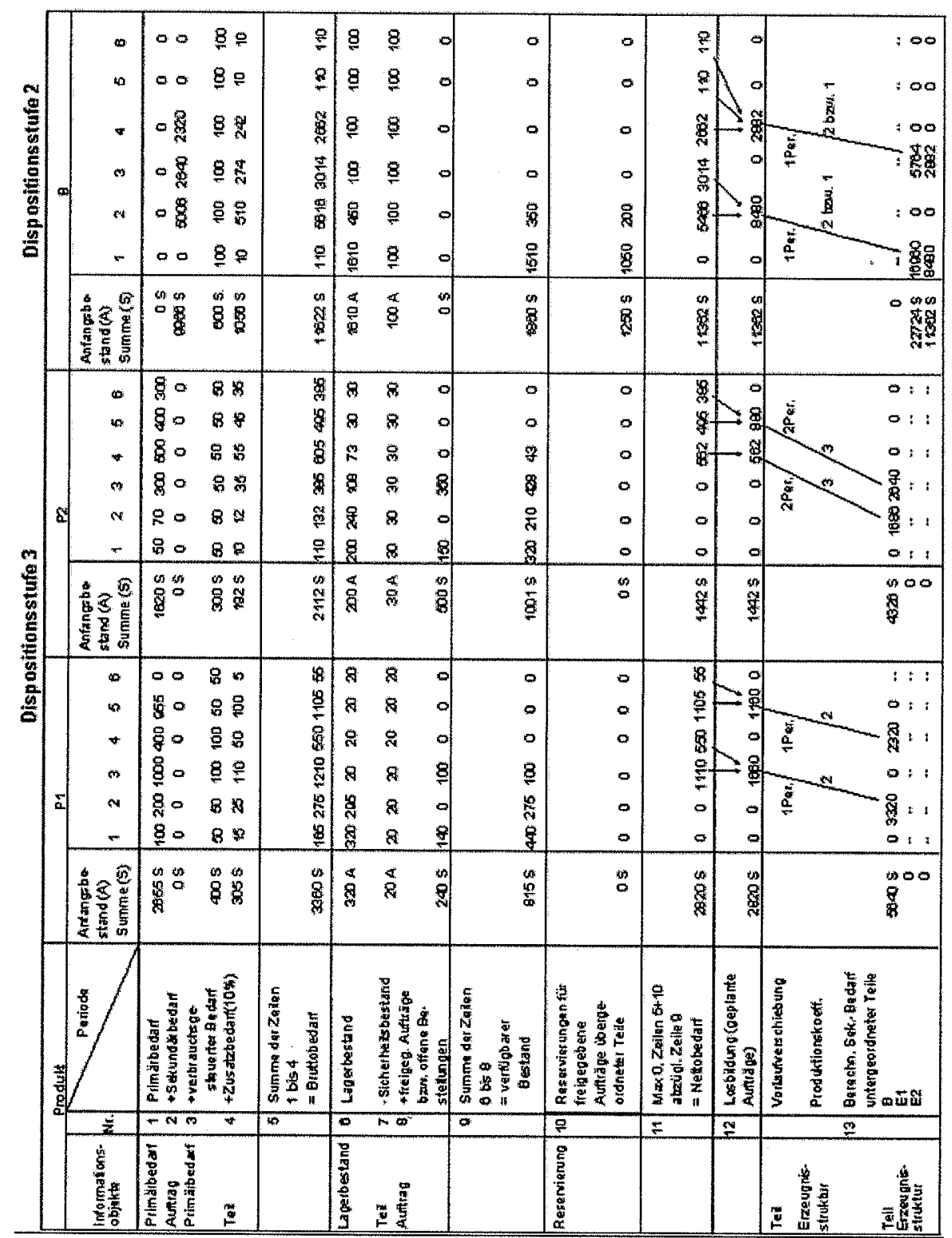

Abbildung 2: Schema der terminierten Bedarfsrechnung [Sche97, S. 142]

Viewlets beziehen sich auf einen Screenshot oder eine Sequenz von Screenshots und erlauben es, bestimmte Bildschirminhalte zu erläutern bzw. zu kommentieren. Texte können kontextspezifisch platziert werden. Vgl. Qarbon: Viewlets. Effective and intuitive online presentation media. http://www.qarbon.com/products/viewlet/ 


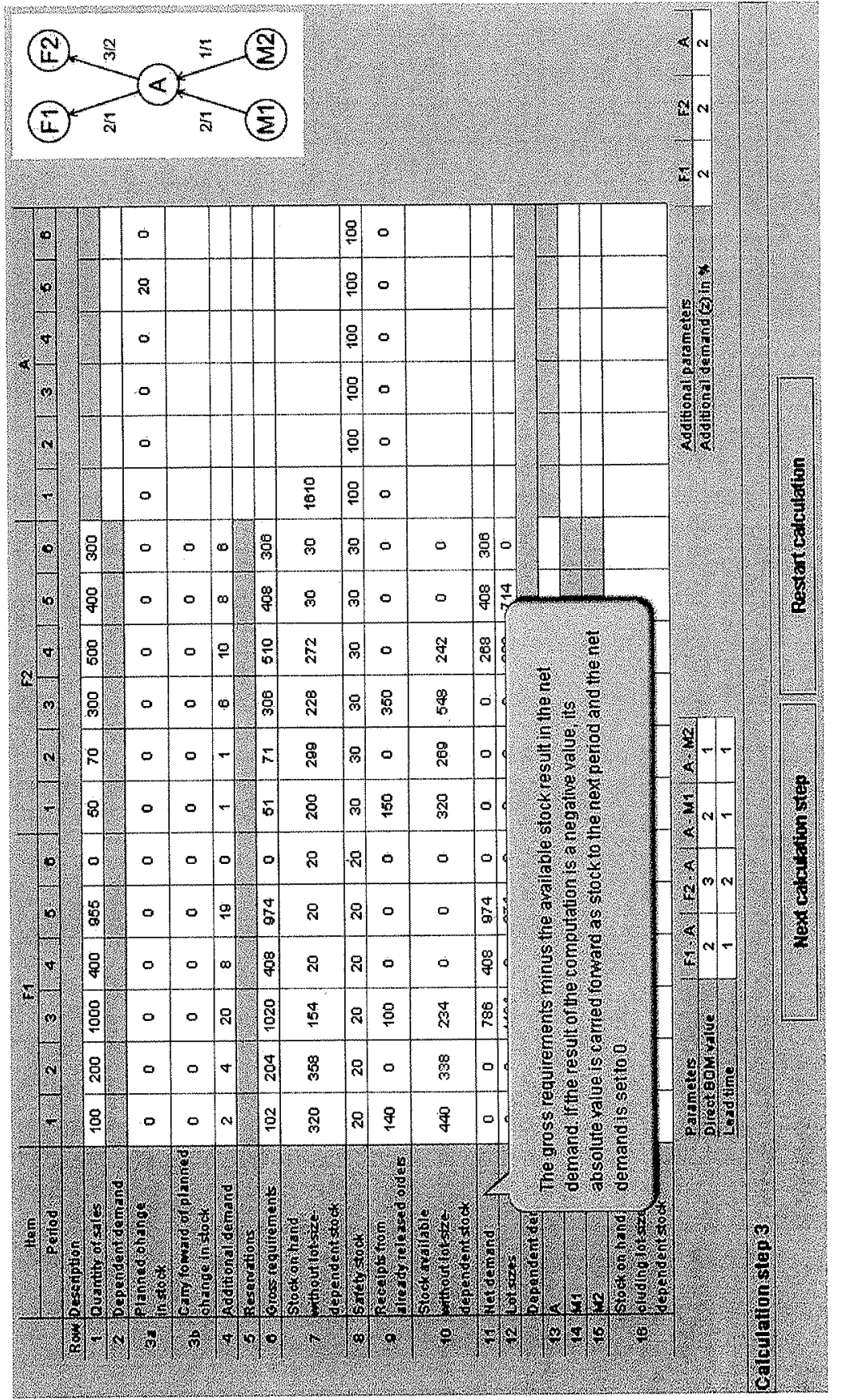

Abbildung 3: Modifiziertes Schema der terminierten Bedarfsrechnung im MLO und Erläuterung der Berechnung des Nettobedarfs mittels Viewlet-Technologie

\subsection{Multimediales Lernobjekt 3: Ziele in der Ablaufplanung}

In der Bestimmung der Reihenfolgen, in der einzelne Fertigungsaufträge auf verschiedenen Anlagen bearbeitet werden sollen, treten häufig Konflikte zwischen der Erreichung logistischer Ziele auf. Oft werden mehrere Ziele der Ablaufplanung formuliert [vgl. z. B. Mell66, S. 164, der 27 Zielgrößen zusammenstellt]. Das folgende Beispiel orientiert sich an jenen Zielgrößen, die im LEKIN ${ }^{\circledR}$ - Flexible Job-Shop Scheduling System ${ }^{4}$, einer an der Stern School of Business, New York University, von Michael L. Pinedo entwickelten Multimedia-Anwendung zur Ablaufplanung [vgl. Abschnitt 3.5], verwendet werden.

Abbildung 4 veranschaulicht, wie wir Definitionen von Zielgrößen der Ablaufplanung vermitteln: Der Studierende wird dadurch, dass nicht alle Definitionen gleichzeitig sichtbar sind, nicht überfordert oder demotiviert, sondern kann durch Anklicken einzelner Zielgrößen ihm unbekannte Definitionen abrufen. Damit wird Wissen (Kategorie 1 nach Bloom) vermittelt. Auf diesem Wissen bauen die in Abbildung 4 veranschaulichten Fragen auf, die auf mit Hilfe der LEKIN ${ }^{\circledR}$-Software erstellten Gantt-Diagrammen basieren.

Würden wir danach fragen, warum die einzelnen Zielgrößen zu unterschiedlichen Ablaufplänen führen, wäre ein vertieftes Verständnis der Zusammenhänge für die Beantwortung erforderlich ("Comprehension" im Sinn von Bloom). Fragt man im Beispiel nach der "Makespan", so kann diese direkt aus dem Gantt-Chart abgelesen werden; wir interpretieren dies als bloße Wiedergabe von Wissen. Fragen wir hingegen nach der "Total Weighted Flow Time", so ist diese aus Abbildung 4 nicht direkt ablesbar. Der Studierende muss zur Beantwortung der Frage die Summe

$(2 * 27)+(2 * 17)+(1 * 34)=122$

berechnen, was im Sinne der Taxonomie von Bloom als Anwendung von Wissen der Klasse 3 zugeordnet werden kann.

\subsection{Multimediales Lernobjekt 4: Belastungsorientierte Auftragsfreigabe}

Die Belastungsorientierte Auftragsfreigabe ist eine Heuristik, die trotz einiger konzeptioneller Schwächen [vgl. Knol91] in der Praxis (vorübergehend) erhebliche Aufmerksamkeit gefunden hat. Bei Anwendung dieses Verfahrens kann der Planer durch Setzung von Parametern die Zahl freigegebener Aufträge beeinflussen [Wien87]. Die zu Grunde liegenden Berechnungen sind für viele Studierende schwierig verständlich.

\footnotetext{
http://www.stern.nyu.edu/om/software/lekin/
} 


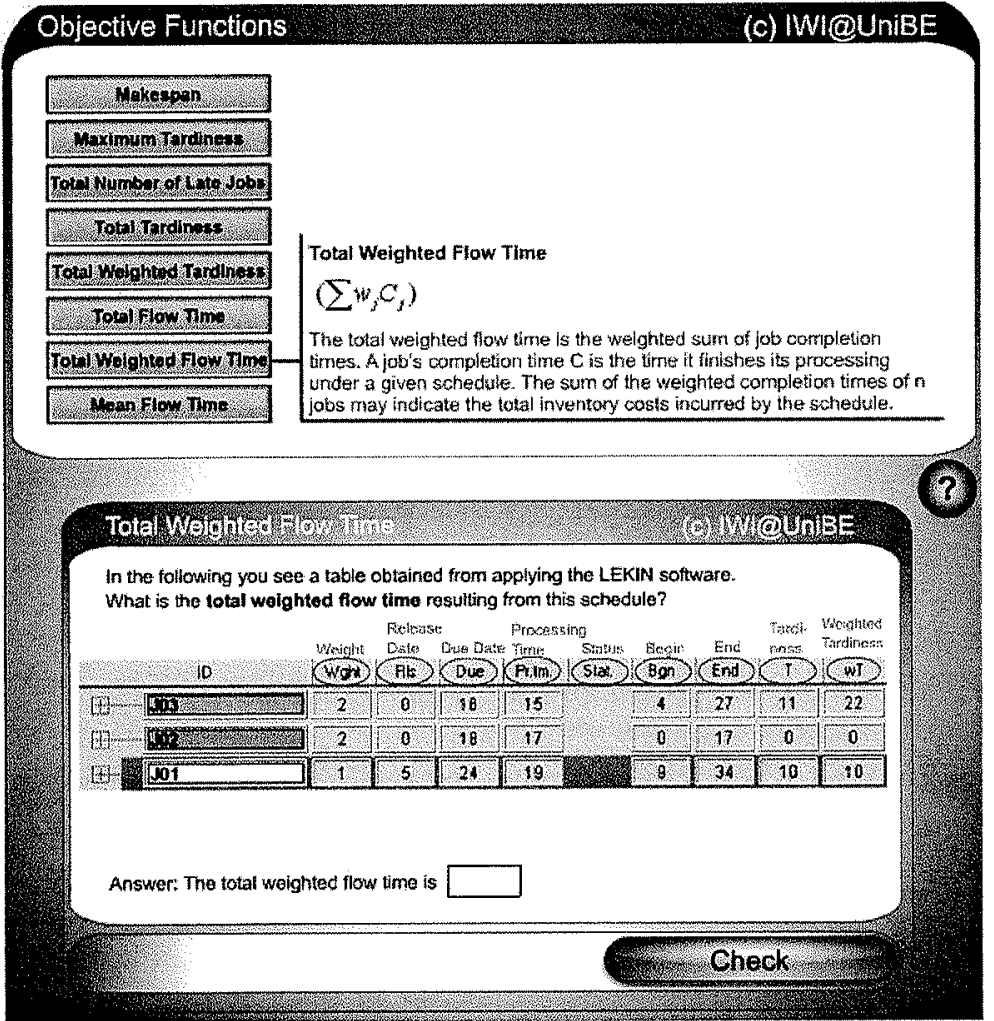

Abbildung 4: Dynamische Bereitstellung von Definitionen und deren Anwendung zur Beantwortung von Fragen nach dem Wert ausgewählter Zielgrößen der Ablaufplanung

Mit dem in Abbildung 5 dargestellten MLO erlauben wir dem Lernenden, die Planungssituation zu spezifizieren: Er kann

- zwischen 1 und 6 Arbeitsgänge für den von ihm betrachteten Fertigungsauftrag definieren

- die auf drei Ressourcen verfügbaren (Rest-)Kapazitäten angeben und

- die Belastungsschranke (Load Limit, LL) und den Einlastungsprozentsatz (Load Percentage, LP) festlegen.

Die entsprechend der Einplanungsheuristik abgewerteten Kapazitätserfordernisse der einzelnen Arbeitsgänge werden im MLO unmittelbar nach Eingabe der Arbeitsgangdaten auf den Belastungskonten der drei Maschinen X, Y und Z in Farbe dargestellt. Die Rechnung und die Visualisierung der Ergebnisse werden unabhängig davon weitergeführt, ob das Verfahren zu einer Freigabe des Auftrags führen wird oder nicht. Dieser Teil des MLO unterstützt die Kategorien "Knowledge" und "Comprehension".

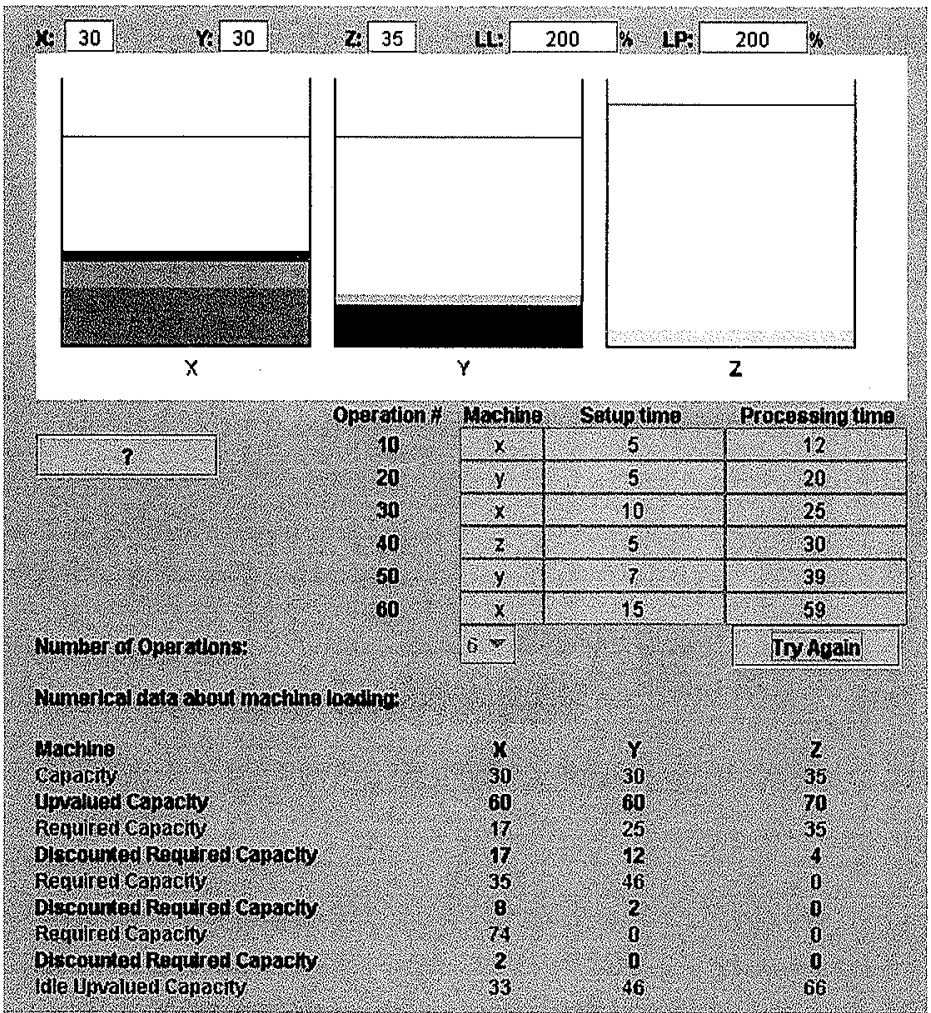

Abbildung 5: Screenshot aus dem Applet zur Belastungsorientierten Auftragsfreigabe

Im zweiten Teil dieses MLO wird der Studierende mit drei verschiedenen Planungssituationen konfrontiert. Für jeden dieser Fälle soll das Applet angewendet werden. Der Studierende muss für jede der drei Situationen beurteilen, ob der Auftrag freigegeben werden soll oder nicht. Er muss zudem erkennen, welche der drei beschriebenen Situationen vorliegt (vgl. Abbildung 6).

Für die Freigabeentscheidung ist die genaue Formulierung des Freigabekriteriums von Bedeutung. Wiendahl [Wien87, S. 213] formuliert: "Sobald durch die Belastung eines Arbeitsganges die Belastungsschranke des zugehörigen Kontos erstmals überschritten wird, wird dieses Konto gesperrt." In unserem MLO, in dem jeweils nur 1 Auftrag betrachtet wird, kann diese Situation dadurch eintreten, dass eine bestimmte Maschine für zwei oder mehrere Arbeitsgänge dieses Fertigungsauftrags benötigt wird. Dies trifft in Abbildung 6 im dritten Fall für Ressource $Z$ zu.

Wir ordnen diesen Teil des MLO den Kategorien "Knowledge", "Comprehension" und "Application" zu. Da die Ergebnisse mehrerer Anwendungen verglichen und 
in einen größeren Kontext gestellt werden müssen, unterstützt das MLO zudem das Lernziel "Analyse".

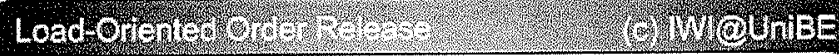

The three different situations that can occur when applying the load-oriented order release method.

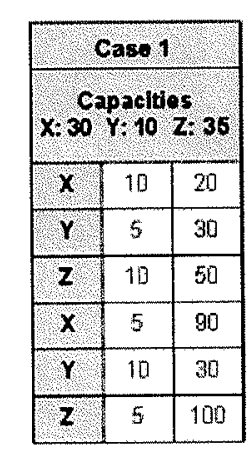

Situation

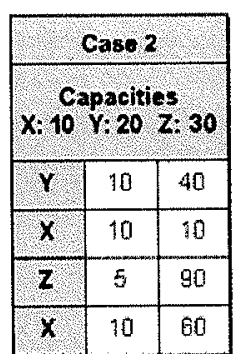

\begin{tabular}{|c|c|c|}
\hline \multicolumn{3}{|c|}{ Cass 3} \\
\hline \multicolumn{3}{|c|}{ Capactios } \\
X: 30 Y: 10 & Z: 36 \\
\hline$Z$ & 20 & 55 \\
\hline$X$ & 10 & 60 \\
\hline$Y$ & 15 & 60 \\
\hline$Z$ & 10 & 20 \\
\hline
\end{tabular}

Situation

Situation
Situation 1: The capacities of all machines are not overloaded even after assigning this order.

Situation 2: The capacity limil of at least one machine is exceeded by charging the cliscounted operation time. However, the order can be releanged because no subsequen
bottleneck machine.

Siluation 3: Because an already overloaded machine is demanded by an operation, the order cannot be red
assignments have to be revoked.

2.

-2. Check

Abbildung 6: Fallunterscheidungen bei der Belastungsorientierten Auftragsfreigabe

\subsection{Multimediales Lernobjekt 5: Reihenfolgeplanung}

Eines der am intensivsten untersuchten Probleme der Produktionslogistik ist die Bestimmung geeigneter Reihenfolgen, in der Fertigungsaufträge auf bestimmten Anlagen ausgeführt werden sollen. Dafür wurden zahlreiche Heuristiken entwickelt, die zu unterschiedlichen Zielerreichungen führen. Die Heuristiken können automatisch angewandt werden. Vielfach werden aber Dialogsysteme oder durch Heuristiken unterstützte Decision-Support-Systeme vorgezogen. Dabei ist es nicht einfach, einen Überblick über die Konsequenzen verschiedener Reihenfolgen für die Erreichung der Zielgrößen zu behalten.
In den Abbildungen 7 bis 9 stellen wir ein Java Applet dar, das auf einer Arbeit von Christoph Burkhalter [Burk o.J.] für educETH.ch ${ }^{5}$ aufbaut. In dem im Rahmen von OPESS weiterentwickelten MLO hat der Lernende die Möglichkeit, bis zu 5 Fertigungsaufträge zu definieren, die bis zu 5 auf verschiedenen Maschinen auszuführende Arbeitsgänge umfassen können. Die Fertigungsaufträge werden im MLO farblich unterschieden. Bereitgestellt wird eine auf die Minimierung der Zielgröße "Makespan" (also die für die Abarbeitung des Auftragbündels benötigte Dauer) ausgerichtete und ein- oder mehrstufig einsetzbare Heuristik; ihre Beschreibung erscheint im MLO nach Anklicken des Help-Buttons. Der Studierende kann aber auch im Dialog, ähnlich wie an einem elektronischen Leitstand [vgl. z. B. ScHa91; Kurb94], durch Drag\&Drop die Reihenfolgen der Arbeitsgänge verändern. Im letzteren Fall passt das Applet die Ablaufpläne automatisch an oder macht auf die Unzulässigkeit der vorgenommenen Änderungen durch Blinken der zugehörigen Balken aufmerksam.

Wir veranschaulichen die Funktionsweise des MLO an den aus Abbildung 7 ersichtlichen Daten. Im Beispiel werden 5 Aufträge mit je 5 Arbeitsgängen betrachtet; Auftrag 3 wird höhere Priorität als den anderen Aufträgen zugewiesen.

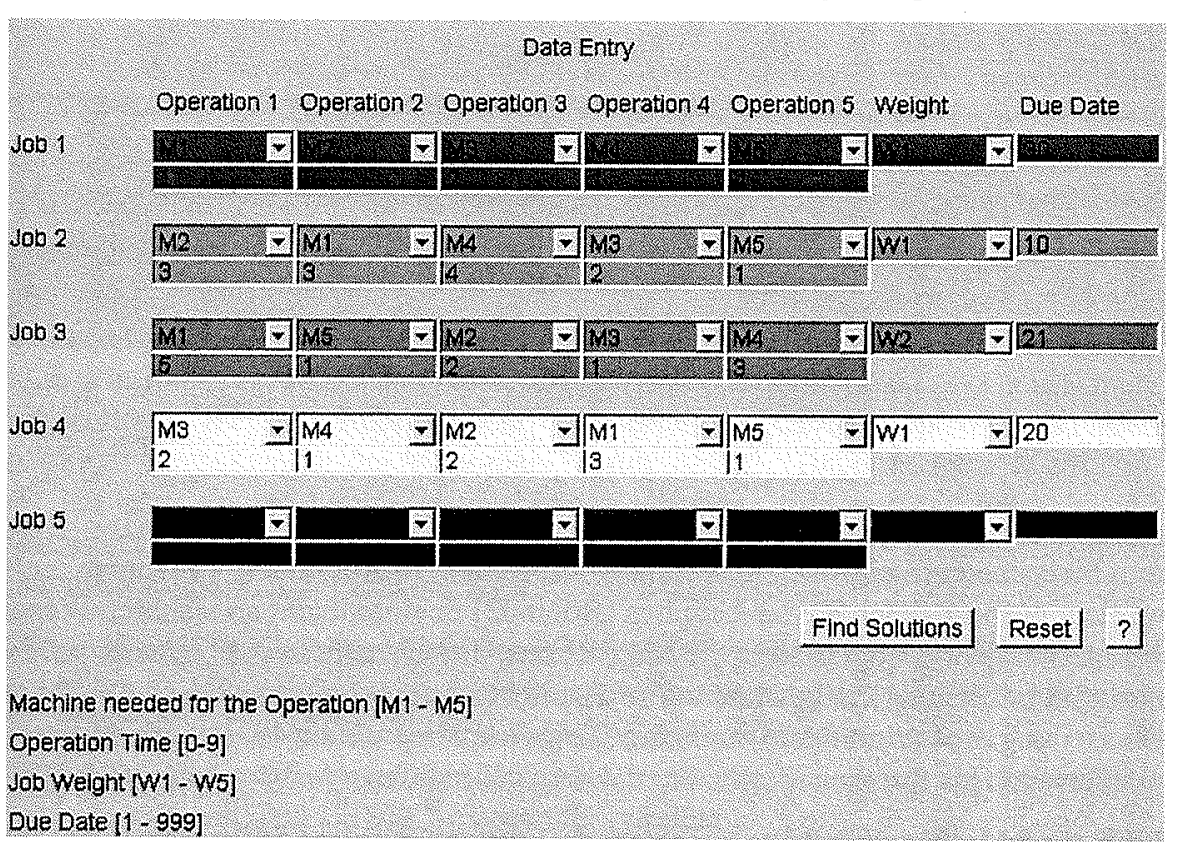

Abbildung 7: Ausgangsdaten für das MLO zur Reihenfolgeplanung 
Im MLO werden die Aufträge und die mit ihnen verbundenen Konsequenzen farblich unterschieden. Die Ausgangslösung plant alle Aufträge nach Auftragsnummern aufsteigend ein; der zugehörige Makespan-Wert beträgt 41. Durch mehrmaliges Auslösen des "Stepwise Improvement"-Buttons reduziert sich dieser Wert sukzessive auf 37, 31, 27, 26, 25 und 22. Abbildung 8 veranschaulicht die zuletzt erhaltene Lösung durch ein maschinenbezogenes Gantt-Diagramm.

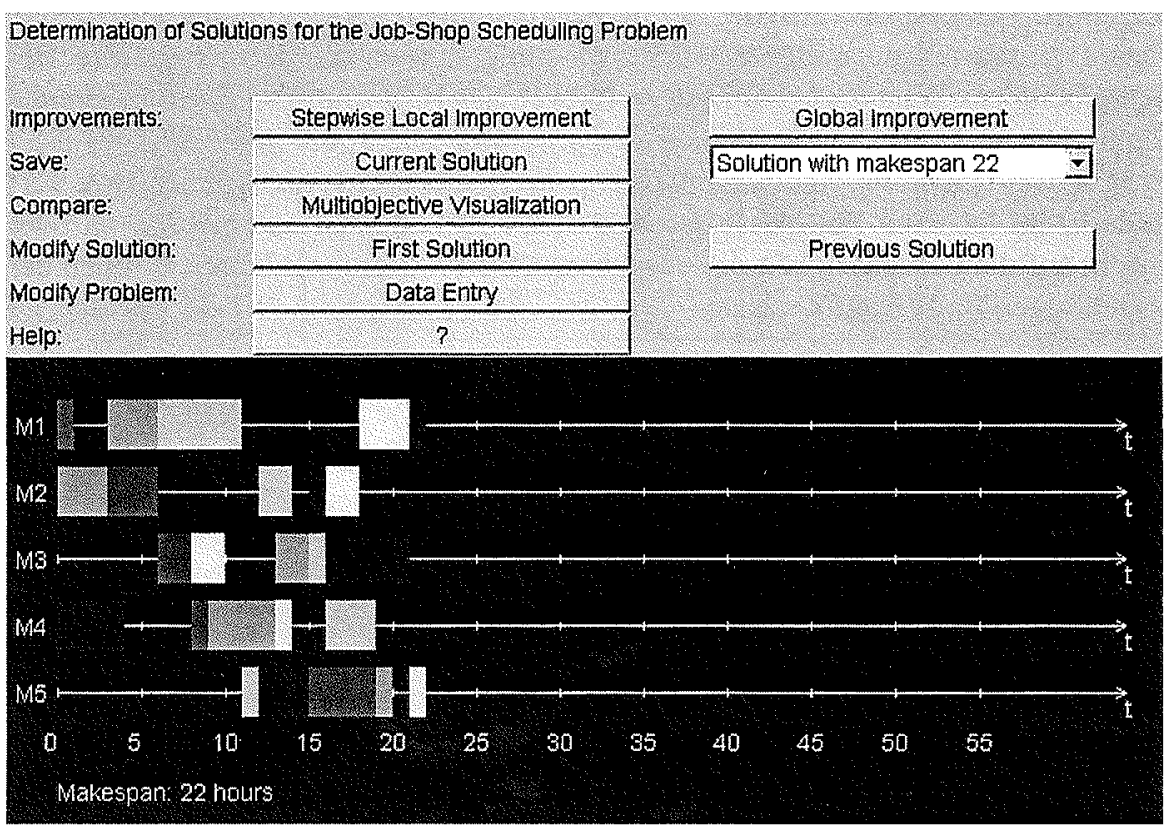

Abbildung 8: Maschinenbezogene Gantt-Diagramme zur besten durch die Heuristik gefundenen Lösung

Für die vier letztgenannten Lösungen haben wir die Werte der 7 im LEKIN $^{\circledR}$-System berechneten Zielgrößen gespeichert; sie werden in Abbildung 9 in einem zielerreichungsbezogenen Kiviat-Graphen [Böhm02] in einer leicht erfassbaren Weise veranschaulicht. Dabei besteht die Möglichkeit, zwischen Lösungen zu unterscheiden, die bei keiner Zielgewichtung in Betracht kommen, und solchen, für deren Beurteilung eine (zumindest implizite) Zielgewichtung unumgänglich ist. Letztere werden auch als funktional effiziente Lösungen bezeichnet [ChCo61, S. 321].

Im Beispiel liefert die zweite gespeicherte Lösung (mit dem Makespan-Wert 26) im Hinblick auf keine der 7 Zielgrößen ein schlechteres Ergebnis als die erste Lösung (mit dem Wert 27). Die dritte Lösung (mit dem Wert 25) wäre nicht in der Lage gewesen, Lösung 1 auszuschließen, weil sie im Hinblick auf mehrere Zielgrößen schlechter abschneidet als Lösung 1. Die vierte gespeicherte Lösung (mit dem Makespan-Wert 22) erlaubt es, die Lösung mit dem Wert 25 als ineffizient zu eliminieren. Hingegen kann die zweite Lösung (mit dem Makespan-Wert 26) nicht ausgeschlossen werden, da mit dieser der geringste Wert für die maximal auftretende Lieferterminüberschreitung verbunden ist. Funktional effizient sind somit bei Betrachtung der gespeicherten Reihenfolgen die (im MLO blau und gelb dargestellten) Lösungen 2 und 4.

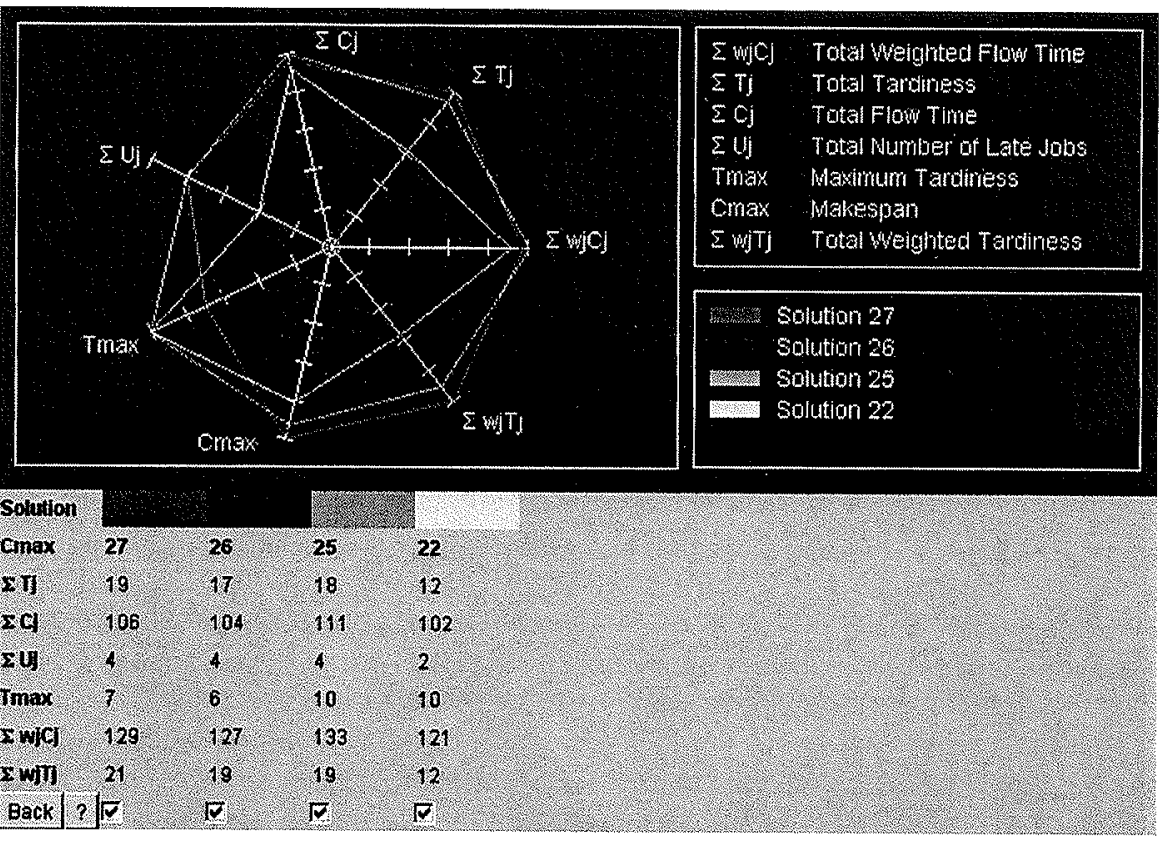

Abbildung 9: Veranschaulichung der Zielerreichungen durch vier Ablaufpläne

Ein weiteres "Stepwise Improvement" oder auch das "Global Improvement" führt $\mathrm{zu}$ keiner Verbesserung des Makespan-Wertes von 22. Hingegen kann es im Dialog mit dem Applet durch Verschiebungen einzelner Arbeitsgänge gelingen, die Makespan auf den Wert von $17 \mathrm{zu}$ reduzieren. Die implementierte Heuristik liefert also nicht immer den minimalen Wert für die Makespan.

Gibt man dem Studierenden eine Datenkonstellation vor, in der die Heuristik nicht die optimale Lösung findet, so kann er nach Anwendung der Heuristik aufgefordert werden, im Dialog nach Verbesserungsmöglichkeiten zu suchen. Als Anreiz für diesen Suchprozess kann der Minimalwert für die Makespan angegeben werden, der z. B. mit dem LEKIN ${ }^{\circledR}$ - System ermittelbar ist.

Im Sinn der Taxonomie von Bloom deckt dieses MLO alle sechs Kategorien ab, weil auch das Problem der Bewertung von Lösungen im Hinblick auf konkurrierende Zielgrößen und Auswirkungen der Methodenwahl thematisiert werden. 


\section{6 Übersicht über die Zuordnungen von multimedialen Lernobjekten zu den in Blooms Taxonomie formulierten Lernzielen}

Die Zuordnung der oben dargestellten MLO zu der von Bloom et al. entwickelten Taxonomie von Lernzielen veranschaulichen wir in Abbildung 10. Nicht in allen Fällen erscheint uns diese Zuordnung als unstrittig. Da für alle und insbesondere auch die anspruchsvollsten Klassen in Blooms Taxonomie multimediale Objekte gefunden wurden, welche die jeweiligen Lernziele unterstützen, kann davon ausgegangen werden, dass mit multimedialen Lernobjekten Beiträge zur Erreichung aller Lernziele möglich sind. Allerdings ist zu berücksichtigen, dass der Spezifikations- und Implementierungsaufwand für MLO, die höheren Klassen der Bloomschen Taxonomie zuzuordnen sind, typischerweise deutlich höher ist als jener für die Erstellung von Objekten, die weniger anspruchsvolle Ziele erreichen sollen.

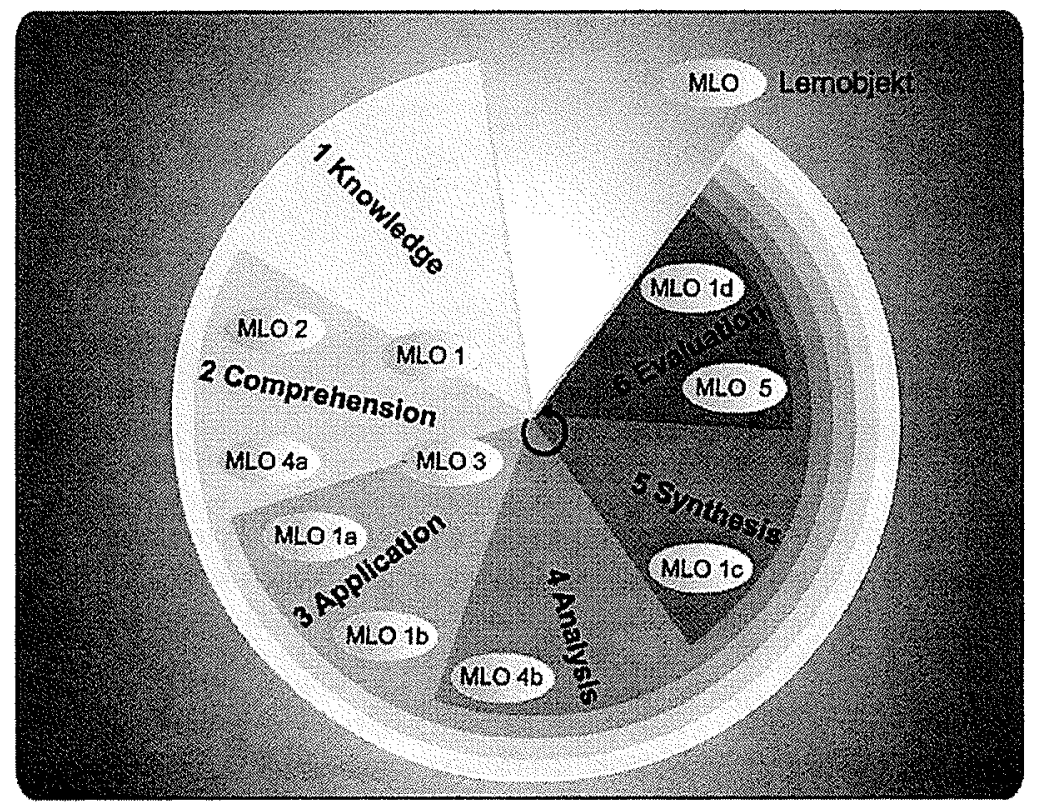

Abbildung 10: Übersicht über die Zuordnung der multimedialen Lernobjekte zu den in Blooms Taxonomie unterschiedenen Lernzielen

\section{Zusammenfassung und Ausblick}

In diesem Beitrag skizzierten wir die Taxonomie von Bloom, die sechs Klassen von Lernzielen unterscheidet. Anhand dieser Taxonomie klassifizieren wir am Institut für Wirtschaftsinformatik der Universität Bern entwickelte und u.a. in Kursen der Virtual Global University eingesetzte Multimedia-Lernobjekte ${ }^{6}$ und zeigen, dass diese für die Unterstützung unterschiedlicher Typen von Lernzielen geeignet sind. Insbesondere existieren multimediale Lernobjekte wie MLO 1d und MLO 5, welche zur Erreichung aller sechs Klassen von Lernzielen beitragen.

Wir haben in diesem Beitrag die Taxonomie von Bloom unter anderem deshalb thematisiert, weil wir uns des Eindrucks nicht erwehren können, dass im Bereich des e-Learnings das Rad ohne Beschäftigung mit den in der Pädagogik erarbeiteten Erkenntnissen teilweise neu erfunden wird. Ein Indiz für diese Vermutung ist auch die Tatsache, dass die in der Elaboration Theory entwickelten Grundsätze [vgl. z. B. Reig79; Reig92] für die Sequenzierung von Lernobjekten in e-Learning-Angeboten weitgehend unbeachtet geblieben sind. In den Projekten OPESS und jenen der Virtual Global University ${ }^{7}$ versuchen wir, bei Entwicklung von e-Learning-Angeboten Fachwissen unter Berücksichtigung gesicherter pädagogischer Erkenntnisse multimedial umzusetzen.

\section{Literatur}

[Bloo $\left.{ }^{+} 56\right]$ Bloom, B.S.; Engelhart, M.D.; Furst, E.J.; Hill, W.H.; Krathwohl, D.R.: Taxonomy of Educational Objectives, The Classification of Educational Goals, Handbook I: Cognitive Domain. New York: McKay 1956, reprinted 1969.

[Böhm02] Böhm, T.: Logiscope-Intro. http://ivs.cs.uni-magdeburg.de/ lother/Lehre/ Foliensaetze/Tut_Logiscope_AuditRulechecker/, 2002, Abruf am 2003-05-13.

[Burk o.J.] Burkhalter, C.: Einleitung in das Job-Shop Scheduling. http://www.educeth.ch/informatik/interaktiv/scheduling/einleitung.html, o.J., Abruf am 2003-05-13.

[ChCo61] Charnes, A.; Cooper, W.W.: Management Models and Industrial Applications of Linear Programming, Volume 1, New York et al.: Wiley 1961.

[GrMe97] Grauer, M.; Merten, U.: Multimedia. Entwurf, Entwicklung und Einsatz in betrieblichen Informationssystemen. Berlin et al.: Springer 1997.

Die MLO sind unter http://www.ie.iwi.unibe.ch/wi2003/verfügbar. http://opess.ie.iwi.unibe.ch/; http://www.vg-u.de/ 
[Knol91] Knolmayer, G.: A Widely Acclaimed Method of Load-Oriented Job Release and Its Conceptual Deficiencies, in: Fandel, G., Zäpfel, G. (Ed.): Modern Production Concepts, Berlin et al.: Springer 1991, S. 219-236.

[Knol03] Knolmayer, G.: Decision Support Models for Composing and Navigating through e-Learning Objects, in: Sprague, R.H. (Ed.): Proceedings of the 36th Annual Hawaii International Conference on Systems Sciences (HICSS-36), Los Alamitos et al.: IEEE 2003, S. 31 and CD-ROM.

[Kurb94] Kurbel, K.: Improving Short-term Production Scheduling and Control by Multimedia Features - An Object-oriented Leitstand Approach, in: Chinese University of Hong Kong (Ed.): Proceedings of the International Conference on Data and Knowledge Systems for Manufacturing and Engineering, Vol. 2, Hong Kong 1994, S. 716-721.

[Kurb99] Kurbel, K.: Produktionsplanung und -steuerung - Methodische Grundlagen von PPS-Systemen und Erweiterungen, 4. Aufl., München-Wien: Oldenbourg 1999.

[Mel166] Mellor, P.: A Review of Job Shop Scheduling, in: Operational Research Quarterly 17 (1966) 2, S. 161-171.

[Mert01] Mertens, P.: Integrierte Informationsverarbeitung 1, Operative Systeme in der Industrie, 13. Aufl., Wiesbaden: Gabler 2001.

[Reig79] Reigeluth, C.M.: In search of a better way to organize instruction: The elaboration theory, in: Journal of Instructional Development 2 (1979) 3, S. 35-40.

[Reig92] Reigeluth, C.M.: Elaborating the Elaboration Theory, in: Educational Technology Research \& Development 40 (1992) 3, S. 80-86.

[Sche97] Scheer, A.-W.: Wirtschaftsinformatik, Referenzmodelle für industrielle Geschäftsprozesse, 7. Aufl., Berlin et al.: Springer 1997.

[ScHa91] Scheer, A.-W.; Hars, 'A.: The Leitstand - A New Tool for Decentral Production Control, in: Fandel, G.; Zäpfel, G. (Eds.): Modern Production Concepts. Berlin: Springer 1991, S. 370-385.

[Schu02] Schulmeister, R.: Grundlagen hypermedialer Lernsysteme, Theorie - Didaktik Design, 3. Aufl., München-Wien: Oldenbourg 2002.

[Thib01] Thibault, M.: Bloom's taxonomy in action ... online http://www.learnnc.org/index.nsf/doc/bloom0405-2, 2001, Abruf am 2003-05-13.

[Vazs54] Vazsonyi, A.: The use of mathematics in production and inventory control, in: Management Science 1 (1954) 1, S. 70-85.

[WaRa99] Warren, K.J.; Rada, R.: Manifestations of Quality Learning in Computer-Mediated University Courses, in: Interactive Learning Environments 7 (1999) 1, S. 57-80.

[Wien87] Wiendahl, H.P.: Belastungsorientierte Fertigungssteuerung - Grundlagen, Verfahrensaufbau, Realisierung, München-Wien: Hanser 1987.

[Wile02] Wiley, D.A. (Ed.): The Instructional Use of Learning Objects, Bloomington: Agency for Instructional Technology and the Association for Educational Communications and Technology 2002. 